\title{
Study on the Path Optimizing Countermeasures of Hengyang National Innovative City Construction under the Background of Rural Revitalization
}

\author{
Bu Huabai ${ }^{1}$, Bu Jiaqi $^{2} \&$ Liu Xinyao ${ }^{1}$ \\ ${ }^{1}$ Hengyang Normal University, China \\ ${ }^{2}$ Krirk University, Thailand \\ Correspondence: E-mail: Bu Huabai, Hengyang Normal University, Hengyang 421008, Hunan, China. E-mail: \\ buhuabai@163.com
}

Received: July 20, 2020

Accepted: August 26, 2020

Online Published: September 16, 2020

doi:10.5539/ijbm.v15n10p27

URL: https://doi.org/10.5539/ijbm.v15n10p27

\begin{abstract}
The construction of a national-level innovative city is an accelerator for rural revitalization, and the promotion of new kinetic energy for the construction of a national-level innovative city in Hengyang is a systematic project. It is necessary to build an urban innovation ecosystem, urban innovation chain system and urban innovation driving factor system with local characteristics based on its own innovative resource conditions. At the same time, we must cherish the entrepreneurial spirit, strengthen the team of high-tech entrepreneurs, continue to adjust the industrial structure in an orderly manner, focus on promoting the technological upgrading of key industries. Hengyang must speed up the improvement of a series of high-quality city construction science and technology policies and build a complete urban science and technology innovation ecosystem, it is necessary to continuously optimize the coordination and promotion mechanism of achievement transformation and industrialization development, and comprehensively promote the construction of a national-level innovative city in Hengyang City, thereby optimizing the path choice of Hengyang national innovative city construction.
\end{abstract}

Keywords: rural revitalization, national innovative city, path optimizing, countermeasures

\section{Introduce the Problem}

To build an innovative city is to thoroughly implement the spirit of the 19th National Congress of the Communist Party of China, and carry out General Secretary Xi Jin Ping on "respecting the regional agglomeration laws of scientific and technological innovation, exploring differentiated innovation development paths according to local conditions, accelerating the creation of a technological innovation center with global influence". As early as September 2015, the Hengyang Municipal Party Committee and Municipal Government issued the "Opinions on Implementing Innovation-driven Development Strategies to Accelerate the Construction of Innovative Cities" and made a major decision to create a national innovative city in Hengyang. In December 2015, the municipal government established the "Hengyang National Innovative City Pilot Construction Leading Group" headed by the executive deputy mayor, who was fully responsible for this work. In early January 2016, Hengyang City communicated specifically to the Ministry of Science and Technology on the Hengyang plan to promote the pilot work of creating a national innovative city, and invited experts to provide suggestions on how to do a good job in the pilot work of creating a national innovative city in Hengyang. On March 10, 2016, experts from the National Science and Technology Information Institute came to Hengyang to investigate the basic situation of Hengyang city construction. In April 2017, Hengyang City formally submitted an application to the Ministry of Science and Technology for the pilot construction of Hengyang to build a national innovative city. At the same time, the Hunan Provincial Government also sent a letter to the Ministry of Science and Technology to affirm the significance of this work and requested strong support for this work. In August 2017, the Ministry of Science and Technology formally accepted applications from the cities. In December 2017, the Ministry of Science and Technology organized experts to review the creation plans of various regions. In April 2018, the Ministry of Science and Technology and the National Development and Reform Commission jointly issued a letter on supporting 17 cities in 11 provinces (autonomous regions) including Hengyang City to carry out innovative city construction. 2019 and 2020 are the key nodes for Hengyang to build a national-level 
innovative city. This article mainly studies how to quickly upgrade the new kinetic energy of Hengyang's national-level innovative city construction according to its own resource conditions under the Background of Rural Revitalization.

\section{Literature Review}

The construction of innovative cities is a hot spot in current theoretical research and practical research. Different experts and scholars have different understandings on how to build a national innovative city. Looking at the relevant literature, it can be found that Schumpeter, an American economist, first proposed the "innovation" doctrine. He believes that innovation includes organizational innovation, product innovation, market innovation, technological innovation, and resource allocation innovation. Charles Landry (founder of Comedia, an authoritative research institution for innovative cities in the UK) believes that the construction of innovative cities not only sounds the horn that promotes the rapid improvement of the quality of urban life, but also provides a toolbox for cities to implement recovery.

Hospers believes that an innovative city is the place where the knowledge economy is bred, urban innovation is an inevitable requirement of the knowledge economy, and a vibrant city must also be an organic combination of good reputation, diversity, agglomeration and instability. Landry (2008) completed the first book on innovative city construction, arguing that inspiring residents to participate in innovative city construction requires certain conditions, such as stimulating innovation potential. James (2013) based on the relevant data of five European innovative cities, studied the main factors affecting urban innovation, and found that the four aspects of urbanization economy, urban internal efficiency, globalization effect, and enterprise spatial agglomeration relationship affect the city innovation.

In China, the National Innovation System Construction Strategy Research Group believes that the connotation of innovative cities has a three-fold structure, that is, to cultivate urban innovation development capabilities, choose to implement urban innovation development models, and focus on urban innovation development achievements. Other scholars generally start from four perspectives: driving force of urban development, the development and evolution of the city, the urban construction system and the mechanism and goals of urban development. It is believed that an innovative city is a city development model in which human capital and knowledge (including science and technology) are the core innovation driving factors, it is a product of the development and integration of the urban economy and the knowledge economy, or a complex urban innovation system that gathers and allocates innovative resources so that the goals of various stakeholders and the urban development goals are aligned.

For how to build an innovative city, Tang Jin (2009, pp. 88-96), Qian Wei (2017, pp.16-19), and Wang Chengwei (2011, pp. 634-640) studied the construction of innovative cities based on the experience of Japan, the United States, and India, respectively. Wen Xiaoling (2016, pp.46-48), Hu Yu (2017, pp. 32-38) et al. proposed the Beijing model, Shanghai model, Shenzhen model and Dalian model; Wang Wei et al. (2018) took Shijiazhuang and Tangshan as examples to study the experiences and practices of the two cities in building innovative cities; Lixing, Zhang Min (2019, pp. 32-34) explored the path of differentiated and innovative city construction, and proposed the strategic positioning Wuhu to build a dynamic innovation and pioneering area in the Yangtze River Delta region, an attractive innovation element cluster in the central region, and an influential industrial innovation center in the Yangtze River Economic Belt. He Shuo (2019, pp. 68-76+103) used the innovation policy as the independent variable and the city's comprehensive innovation index as the dependent variable, and used the city dynamic panel system GMM estimation method to demonstrate the innovation policy of the local government in the innovation city pilot construction about the city's innovation ability and driving effect. The results of the study show that: the policy of scientific and technological talents and industrial policies have a significant role in promoting the innovation capacity of innovative cities; Wang $\operatorname{Li}(2019$, pp. 78-85)believes that innovation subjects, innovation systems, innovation resources and the support system of innovation culture are the core in the construction of innovative cities, the integration of innovation elements can enhance the cooperation of government, industry, research and research platforms, and achieve the collaborative innovation effect of integrating system innovation, industrial innovation, knowledge innovation, and technological innovation; Zhang Xiangyang (2019, pp. 52-54) believes that many cities in China are in a critical period of development and transformation. Under the new situation of increasingly fierce international competition, urban development urgently needs to solve the problem of economic development and industrial structure imbalance, extensive production methods and independent innovation insufficient capacity, etc.

In short, with the in-depth adjustment of the global industrial chain and innovation chain and the emergence of a new pattern of industrial transformation and development, many urban development models with innovation as 
the core driving force have been tested by theory and practice. From the perspective of the development and evolution of innovative cities, innovative cities are generally evolved from regional science and technology centers. Their economic growth model is formed by the aggregation and allocation of elements in innovative resources, innovative environment, and innovative institutions. The model will have an innovative radiation and leading role in other surrounding areas.

\section{Factors Affecting the Path Choice of Hengyang National Innovative City Construction}

The factors driving the improvement of new kinetic energy in the construction of innovative cities are not only the basic support and important components of urban innovation resources, but also the elements of urban aggregation and allocation of innovation. They are the driving force that stimulates the city's innovation function and drives economic and social development, including innovative talents ( Entrepreneurs, research and development talents, experts and scholars, front-line employees, etc.), innovative investment (funds, manpower, land, etc.), innovative technology (knowledge information, technological innovation, business model, etc.), innovative institutions (government, enterprises, universities and scientific research Institutions, financial institutions, etc.) and government support (infrastructure, macro-policy, social environment, etc.). In the following, we will specifically analyze the advantages and bottleneck factors that affect the new kinetic energy of the construction of Hengyang's national innovative city.

\subsection{Advantageous Factors Affecting the Path Choice of Hengyang National Innovative City Construction}

3.1.1 Hengyang's Innovative City Construction Has a Solid Foundation and Obvious Advantages in Innovation Resources

As a historical and cultural city, Hengyang has a solid foundation in the construction of a state-level innovative city, and has obvious advantages in innovation resources, which are embodied in the following five aspects.

(1) Superior geographical location

Hengyang is the second largest city in Hunan Province. Located in southern of Hunan, it is the economic and cultural center of southern Hunan, with a total area of 15,310 square kilometers and a permanent population of 8.05 million. Hengyang is located at the south of Hengshan Mountain in Nanyue. It is the confluence of the Xiang River and its tributaries Lei and Distilled Water. The intersection of Beijing-Guangzhou and Xiang-Gui Railways is the water and land transportation center in southern Hunan and a transportation hub connecting north and south.

Located in the Pan-Pearl River Delta Regional Center, Hengyang is known as "the coastal inland, the frontier of the inland". It is the country's 45 main railway and rail transportation hub cities, and 32 important Internet broadband backbone network cities in the country. Hengyang Nanyue Airport has been in operation for three years. The passenger throughput has reached 460,000 passengers, more than 20 routes have been opened, the 1000-ton golden waterway of Xiangjiang River, Jingzhu, Hengzao, Hengda, Hengyan, Hengshao, Henggui, Tanhengxi, Hengyue 8 high-speed highways, Beijing-Guangzhou, Xianggui, Hengchaji and other 9 railways have built a transportation network with complete land, water and air coverage, three-dimensional coverage and extending in all directions.

(2) Profound cultural heritage

Hengyang's history of building a city was 2300 years. It was the land of Yangyue in ancient times. The Warring States belonged to Chu. Five Yue in Chinese, Hengyang has one (Hengshan, Nanyue); the four ancient inventions of ancient China, Hengyang has one (Cai Lun paper); the four major academies of the Song Dynasty, Hengyang has one (Shigu Academy); the two famous materialisms philosopher in the world, Hengyang has one (Wang Chuanshan); New China's top ten marshals, Hengyang has one (Luo Ronghuan). Hengyang has 12 colleges and universities. Hengyang in history is an ancient cultural city, a military center, and a commerce and trade town in southern Hunan. It is the hometown of Wang Chuanshan, Cai Lun, and Luo Ronghuan.

(3) Advantages of urban platforms

It has the sub-central cities of Hunan Province, the central cities of the southern Hunan region, the "2025" pilot city made in China, the national garden city,", the "broadband China" demonstration city, and the important industrial cities in central and southern regions. National service industry comprehensive reform pilot cities, national undertaking industrial transfer demonstration zones and other platforms, with national-level high-tech zones, national agricultural science and technology parks, Hunan's first comprehensive bonded zone, At the same time, it is also an advanced city for national scientific and technological progress, a national pilot city for 
intellectual property rights, and leaders of successive municipal Party committees and municipal governments in Hengyang attach great importance to scientific and technological innovation.

(4) Urban industry advantages

Hengyang has the reputation of "hometown of non-ferrous metals" and "hometown of non-metals". It is one of the 26 old industrial bases in the country. It has a complete range of industries, including nonferrous metallurgy, power transmission and transformation manufacturing, automobiles and parts, and salt halogenation. Industrial clusters have been formed, and strategic emerging industries such as new materials, biomedicine, and electronic information are booming. At present. Hengyang has my country's first and only large-scale uranium hydrometallurgical purification plant. Hengyang's uranium detonated China's first atomic bomb, making important contributions to the success of China's atomic bomb explosion and the development of the nuclear industry. Hengyang Cable connected the heart of the "Shenzhou" spacecraft, and Sinosteel Hengzhong produced China's first new continuous casting machine and China's first roller rig.

(5) Urban science and education advantages and policy advantages

With 11 colleges and universities and 150,000 college students, it provides a solid talent pool for the development of Hengyang. At the same time, the Hengyang Science and Technology Bureau has taken many new incentive measures around key areas such as the reform of the science and technology management system, the promotion of the integration of science and technology and finance, the implementation of preferential tax policies, and the improvement of the talent incentive mechanism, which provide a good guarantee for Hengyang's technological innovation.

3.1.2 Hengyang's Innovative City Has Obvious Advantages in Transportation Location, Numerous Financial Institutions, and High-tech Enterprises Developing rapidly

Hengyang is a populous city in Hunan Province, with large consumer groups and great market potential. At the same time, Hengyang is located in a national transportation hub. The domestic railway, highway, air transportation and water transportation are very convenient, and the logistics cost advantage of the enterprise is obvious. At the same time, the comprehensive economic strength is also strong, with a large number of financial institutions (145). A financial market system in which banks, insurance, securities, microfinance companies, guarantees, and other multi-level institutions coexist and complement each other has formed. Not only that, with the deepening of the construction of pilot cities for comprehensive reform of the national service industry, Hengyang's financial informatization level has improved significantly. The Hengyang Regional Issuance Bank of the People's Bank of China in Hengyang City also covers four cities outside of Hengyang: Chenzhou, Yongzhou, Loudi, and Shaoyang, which greatly enhances Hengyang's financial strategic position and radiation effect, and effectively enhances money supply and management efficiency in the southern Hunan region. The financial institutions also provide favorable funding conditions for the rapid development of high-tech enterprises in the region.

3.1.3 The Innovation Environment of Hengyang's Innovative City Construction Is Constantly Optimized to Maintain and Promote Innovation with a High Degree of Security

With the deepening of the construction of national-level innovative cities in Hengyang City, people's innovative ideas have begun to be generally recognized, and they have begun to use innovative thinking to actively solve development problems. At the same time, the government of Hengyang City continues to increase its propaganda for innovation achievements, innovative enterprises, and innovative entrepreneurs, and the independent innovation models of enterprises and individuals have been widely publicized in the society. Not only that, Hengyang City has also fully stimulated the innovation and entrepreneurial vitality of Hengyang City through measures such as increasing the recognition and reward of Hengyang innovation and entrepreneurs.

Hengyang Municipal Government has provided theoretical support and practical guidance for the idea innovation, system mechanism innovation, and method innovation of Hengyang urban development by continuously promoting the development and prosperity of soft science and Hengyang philosophy and social science. At the same time, in terms of the scientific research academic environment, the government has strengthened the construction of academic ethics and scientific research integrity, creating a relaxed, honest, and harmonious scientific research academic environment. In terms of tolerating failure and advocating a good social atmosphere of innovation, the government has paid more attention stimulate the innovation enthusiasm of the whole society, make Hengyang innovative talents continue to stand out, the economic benefits, social benefits and cultural benefits of innovation have been fully revealed, and the cultural connotation of Hengyang people who have been used for the world and dare to take the lead has also been extremely popular. 


\subsection{The Bottleneck Factors Restricting the Path Choice of Hengyang National Innovative City Construction}

\subsubsection{The Status of Enterprise Innovation Subject is Not Strong}

According to the investigation of our research group, in terms of innovation decision-making, scientific research organization, R\&D investment and achievement application, the main position of enterprise technological innovation needs to be further strengthened. In many high-tech fields, the core technology of enterprises with independent intellectual property rights is also relatively lacking. It is reflected in the following four aspects:

(1) Compared with other leading enterprises such as developed coastal areas, it is more difficult for Hengyang to cultivate enterprises with regional competitiveness. There are two main reasons: First, there are not many listed companies in Hengyang. The innovation motivation of other large enterprises is insufficient. The second is that Hengyang SMEs have a low ability to withstand overall innovation risks due to their own operational capabilities;

(2) Hengyang's ability to absorb technology, absorb and further improve it, and the localization of imported technology, the export capacity is relatively inadequate, and the successful experience of technology introduction, digestion, absorption and innovation is less accumulated.

(3) In the urban innovation practice of Hengyang, many small and medium-sized high-tech enterprises have relatively insufficient willingness to invest in the innovation capacity of the enterprise, which leads to the lack of investment in technology introduction, digestion and re-innovation, resulting in a bad cycle;

(4) Hengyang had actively guided enterprises to increase investment in research and development, and actively promoted the industrialization of major scientific and technological achievements of the company, despite the greater efforts, the results achieved in establishing and improving the exchange platform for imported technology and innovation experience and the introduction of technology digestion and absorption technology information service platform are not very obvious.

3.2.2 There Is a Disconnect Between the Knowledge Chain and the Industrial Chain, and the Closeness of the Combination of Industry, University and Research Is Insufficient

We know that only when the knowledge chain and the industrial chain are highly integrated, and the cooperative innovation mechanism of enterprises and research institutes is relatively stable, will the ability to transform scientific and technological achievements be improved, and the scale and industrialization of scientific research achievements will be realized. Compared with the level of developed regions, Hengyang's knowledge chain and industrial chain are highly integrated, resulting in a weak transformation of overall scientific and technological achievements. At the same time, the transformation of scientific and technological achievements lacks flexibility, and the integration of industry, education and research in the process of industrialization of scientific and technological achievements is still not standardized enough, the mechanism is not flexible, it is easy to miss market opportunities, and ultimately it cannot form a large-scale, industrialized development and application. From the perspective of patents, the total number of patent applications in Hengyang is increasing year by year. The total number of patent applications and the growth rate are ranked in the forefront of the province, but the proportion of invention patent applications in the total number of applications is at a significant disadvantage in the province; the total number of effective invention patents and the growth rate continues to increase, but the advantages in the province are not outstanding; the number of invention patents per 10,000 people, the number of invention patents per 100 million GDP, and the growth rate of ownership have no obvious advantages in the province.

3.2.3 The Effectiveness of the Construction of Innovative Service Intermediary System Is Not Strong, the Role Is Not Enough, and the Service Capacity Is Insufficient

The development of intermediary organizations in Hengyang's achievement transformation is not perfect, and the effective sharing of public innovation information resources is not sufficient. Many scientific research institutions and universities are only relatively actively investing in research and development of science and technology planning projects, but they are not enthusiastic about the commercial transformation of achievements. At the same time, the relationship between the obligations and rights involved in the transformation of innovation achievements by the government, units and individuals is not completely straightened out, etc., which leads to the ineffectiveness of the construction of Hengyang's innovation service intermediary system, insufficient effects and insufficient service capacity. 


\section{Countermeasures for Optimizing the Construction Path of Hengyang National Innovative City}

During the three-year national innovation city construction period in Hengyang, it is necessary to seek key breakthroughs in 7 key indicators: invest in the intensity of R\&D expenditures in the whole society, the proportion of public expenditure on science and technology, the number of invention patents per 10,000 people, the number of high-tech enterprises, the growth rate of regional GDP, and the increase in service industry, value ratio and total labor productivity.

4.1 Cherish the Entrepreneurial Spirit, Strengthen the Team of High-Tech Entrepreneurs, and Enhance the City's Creative Breakthroughs and New Development Momentum

Schumpeter believes that the reason for innovation activities is because of the innovative spirit of entrepreneurs. Entrepreneurs are different from ordinary businessmen and ordinary speculators in society. Getting rich personally is only a small part of their motivation, and the most prominent motivation is "entrepreneurship". Schumpeter believed that "entrepreneurship" includes:

(1) enthusiasm for victory; (2) establishment of a private kingdom; (3) strong will; (4) joy of creation. This spirit is not only the source of power to achieve excellent entrepreneurs, but also the intellectual foundation for creative breakthroughs in economic development. Entrepreneurs are the most scarce resource in the market economy, the soul of enterprise technological innovation activities, and also an important indicator to measure the economic development of a country or a region. In order to solve the development problems in the future, Hengyang must strive to create an outstanding team of entrepreneurs in the changing market competition, give full play to the entrepreneurial spirit, encourage entrepreneurs to increase their investment in innovation, and promote leading enterprises to gradually enter the high end of the value chain and innovation chain. At present, Hengyang has introduced 30 preferential policies for investment promotion, 30 preferential industries policies, and 23 preferential talents policies, and has promulgated the "Interim Measures for the Introduction of Insufficient and Innovative Talents." However, to increase the new kinetic energy for the construction of Hengyang National Innovative City, It is necessary to actively strengthen the team of entrepreneurs and scientific and technological innovation and entrepreneurship, actively promote the development project of scientific and technological talents, and accelerate the gathering of entrepreneurs and scientific and technological talents in Hengyang.

4.2 Orderly Adjust the Industrial Structure, Focus on Promoting the Technological Upgrading of Key Industries, and Do a Good Job in Undertaking the Industry

First, based on Hengyang's existing advantageous and characteristic industries, do a top-level design of industrial structure adjustment, prioritize the layout of strategic emerging industries such as equipment manufacturing and bio-manufacturing with development potential and high added value, and help promote Hengyang's industry to innovation chain and value Chain high-end mobile, enhance Hengyang city industrial innovation momentum. For Hengyang, on the one hand, strengthen the top-level design, deeply implement the decision-making and deployment of the "135" project of innovation and entrepreneurship of the provincial party committee and government, actively integrate into the Yangtze River Economic Belt to the north, and actively integrate into the Guangdong-Hong Kong-Macao Greater Bay Area to the south. "City" is an opportunity to introduce large projects aiming at industry benchmarks and do a good job of undertaking industry transfer. On the other hand, Hengyang should build on its own industrial advantages, implement the " 3311 " project development plan, and strive to build 8 hundreds of billions of industrial clusters: new energy vehicles, non-ferrous metals, fine chemicals, rail transportation, textile and clothing, agricultural and sideline products planting and processing, and global tourism.

Second, integrate Hengyang's urban characteristic innovation resources and realize the integrated development of urban innovation chain and industrial chain. First, we must identify the entry point of Hengyang's technology and industry integration development from the nine major industrial clusters in Hengyang, build a collaborative innovation community of Hengyang's technology industry, comprehensively promote the construction of Hengyang's high-quality innovative science and technology park, and form high-quality scientific and technological transformation results , Further strengthen the division of science and technology and cooperative development of the southern Hunan urban agglomeration centered on Hengyang City, form a brand-new innovative value chain for $\mathrm{R} \& \mathrm{D}$ and transformation of industrial clusters, and promote the formation of a mutually beneficial and mutually beneficial development pattern between the Hengyang technology service system and the scientific and technological innovation chain . 
The second is to connect domestic science and technology parks and high-quality scientific and technological resources, and form an industrialization docking chain with the external scientific and technological resources of the "Yangtze River Economic Belt", "Guangdong-Hong Kong-Macao Greater Bay Area" and "Greater Southern Hunan City Group" in R\&D, experimentation, trading and transformation. To form an internal cycle of high-quality scientific and technological resources and a new pattern of "internal and external linkage".

\subsection{Accelerate the Improvement of a Series of Science and Technology Policies for the Construction of High-Quality Cities and Build a Complete Urban Science and Technology Innovation Ecosystem}

High-quality technological innovation activities are a complete "innovation chain", including at least the following eight factors: incubators, public research and development platforms, venture capital, industrial chains formed around innovation, property rights transactions, market intermediaries, legal services, Logistics platform, etc. At the same time, we know that high-quality technological innovation activities need to be supported by an urban innovation ecosystem formed by four elements of innovative talents, technological innovation policies, innovation culture, and innovation chains in order to achieve good innovation results. Freeman believes (the new Schumpeterian in the theory of national innovation system) that government science and technology policies play an important role in promoting urban technological innovation. The main responsibility of the government is to form complete innovation ecology through the guidance of scientific and technological innovation policies, and then maximize the accumulation of high-quality innovation resources at home and abroad to form the city's ability and achievements of continuous innovation. In response to the current problems of Hengyang's innovation motivation, innovation risks, innovation capabilities, and insufficient financing for innovation, the Hengyang Municipal Party Committee and Municipal Government attach great importance to technological innovation, and have included "innovation development" in the overall strategy of Hengyang's development, and have successively issued "Hengyang City Opinions on Promoting the Combination of Industry, University and Research to Enhance Independent Innovation Ability", "Hengyang City's Decision to Accelerate the Cultivation and Development of Strategic Emerging Industries", "Hengyang Science and Technology Incentive Measures (Revision)", "Opinions on Encouraging and Supporting Independent Innovation of Enterprises", "Decisions on the promotion of industrial development and innovation capacity building in the Hengyang High-tech Industrial Development Zone", and "Opinions on the Implementation of Banking and Financial Institutions to Support the Development of Small and Medium-sized Enterprises" and other policies, It has promoted innovation-driven development strategies in many ways, creating a good atmosphere for the whole society to respect innovation and respect talents from system construction, intellectual support, funding guarantees, and incentive mechanisms.

At the same time, the number of high-tech enterprises in Hengyang has a large advantage (ranking the top in the province). The added value of high-tech industries continues to increase rapidly and ranks high in the province. The value-added of high-tech industries accounts for the proportion of GDP increasing year by year and the advantages in the province are obvious. Hengyang's high-tech industry has become the main battlefield for Hengyang's technological innovation-driven development and has become an important growth pole for Hengyang's economic development.

In short, the promotion of new kinetic energy for the construction of Hengyang's national innovative city is a systematic project, and the enthusiasm and creativity of urban innovation subjects (enterprises, scientific research institutions, financial departments, intermediary institutions, education and training institutions, government departments, etc.) must be fully utilized .It is necessary to give full play to the guiding role of the Hengyang Municipal Government, coordinate and balance the position and interests of enterprises, universities and scientific research institutions in the independent innovation system, gather urban innovation resources, and coordinate the promotion of institutional innovation, scientific and technological innovation, knowledge and cultural innovation and science and technology.The construction of intermediary service system, constantly improve the basic research, applied research and the mechanism of coordinated development between the transformation of achievements and industrialization, only in this way, can we maximize the new kinetic energy for the construction of Hengyang National Innovative City.

\section{Acknowledgements}

This article is co-funded by the subject group project of Hengyang Normal University, "Research on the 'bottleneck' of rural revitalization in Hengyang City and its formation mechanism and resolution strategy "(18XKQ03), Hengyang Teachers College Provincial Platform Open Fund Project "Research on the Network Governance Countermeasures of Hengyang Xiehuan Enterprises" (HJ16K02), Industry-University-Research Project of Hengyang Normal University“Research on Cross-layer Optimization 
and Operation Control of Internet of Things for Large Food Processing Enterprises Based on Agents Modeling Technology and Enterprise Value Net Model", Applied Economics Discipline Platform Project in Hunan Province "Thirteenth Five-Year".

\section{References}

$\mathrm{Hu}$, Y. (2017). Connotation, experience and approach of innovative city construction. China Soft Science, (4), 32-38.

Landry, C. (2008). The Creative City: A Toolkit for Urban Innovatiors.

Li, X., \& Zhang, M. (2019). Research on the strategic positioning of Wuhu City to build an innovative city. Anhui Science and Technology, (08), 32-34.

Qian, W. (2017). Innovative City Development Road — The Experience and Enlightenment of the Transformation of Typical American Cities. Reform and Opening, (2), 16-19.

She, S., Wang, Q., \& Zhang, L. (2019). Evaluation of the innovation driving effect of government innovation policy. Urban Issues, (07), 68-76+103.

Simmie, J. (2013). Innovative Cities. Routledge.

Tang, J. (2009). Ways to build an innovative city --- Experience and inspiration from Kawasaki City, Japan. Shanghai Economic Research, (7), 88-96.

Wang, C. W. (2011). Rethinking innovative cities: Take Bangalore, Silicon Valley, India as an example. Scientific Research, (4), 634-640.

Wang, L., Mao, S. L. (2019). Research on the Construction of Innovative City in Xiong'an New District: A Conceptual Analysis Framework. Journal of Tianjin Administration Institute, 21(04), 78-85.

Wang, W., \& Deng, X. L. (2018). Theoretical and practical research on the construction of innovative cities in Hebei Province. Proceedings of the 6th China Science and Technology Policy and Management Annual Conference.

Wen, X. L. (2016). Exploration of domestic innovative city construction. Frontline, (2), 46-48.

Zhang, X. Y. (2019). Research on countermeasures for the construction of innovative cities in my country. Special Zone Economy, (06), 52-54.

\section{Copyrights}

Copyright for this article is retained by the author(s), with first publication rights granted to the journal.

This is an open-access article distributed under the terms and conditions of the Creative Commons Attribution license (http://creativecommons.org/licenses/by/4.0/). 